\title{
Discrimination morphologique des glands de chênes sessile (Quercus petraea (Matt.) Liebl.) et pédonculé (Quercus robur L.)
}

\author{
J.L. Dupouey ${ }^{1}$ et H. Le Bouler ${ }^{2}$
}

1 INRA, Champenoux, 54280 Seichamps;

2 SRFB Pépinière expérimentale de Guéméné-Penfao, route de Redon, 44290 Guéméné-Penfao, France

(reçu le 12-4-1988, accepté le 22-8-1988)

Résumé - Nous étudions la possibilité de discriminer les glands de chênes sessile et pédonculé d'après leur morphologie. Sept mesures de forme ont été effectuées sur 100 glands prélevés dans 44 provenances de chênes françaises, lors de la glandée de 1984. L'analyse en composantes principales montre une nette distinction entre les deux espèces selon la distance entre le rayon maximum du gland et son apex et, dans une moindre mesure, selon son rayon maximum. Un indice d'espèce basé sur ces 2 mesures permet un taux de reconnaissance de $85 \%$ des glands. Cet indice reste à valider pour d'autres années de glandée et sur un échantillon mieux équilibré.

\section{Quercus robur L. - Quercus petraea (Matt.) Liebl. - gland - morphologie}

\begin{abstract}
Summary - Morphological discrimination between Quercus robur L. and Quercus petraea (Matt.) Liebl. acorns. We study the practical feasibility of discrimination between pedunculate oak (Quercus robur L.) and durmast oak (Quercus petraea (Matt.) Liebl.) using morphological parameters. 44 populations were sampled, during mast crop year 1984, mainly from West, South-West and Central France. Seven shape variables were measured on 100 acorns in each population : radii at different level along the acorn, total length, maximum width and position of this maximum width. Principal components analysis shows a net distinction between the two species, according to the distance between the maximum width and the apex, and, in a lesser extend, to the maximum width. Acorn shape is highly correlated with the percentage of auriculated leaves observed after plantation. The species index, based on acorn shape, permits identification of individual acorns with only $15 \%$ error. It remains to be tested for other years and areas.
\end{abstract}

Quercus robur L. - Quercus petraea (Matt.) Liebl. - acorn - morphology 


\section{Introduction}

Les chênes sessile et pédonculé occupent une place de premier plan dans la forêt française (respectivement $13 \%$ et $18 \%$ des surfaces boisées selon l'Inventaire forestier national). Face à l'augmentation constante des surfaces régénérées par plantation, les pépiniéristes doivent produire un nombre croissant de plants. Or, cette croissance s'accompagne de transferts de glands d'origines très variées avec parfois une mauvaise maîtrise de leur provenance. La confusion existe non seulement entre les origines, mais aussi entre les espèces récoltées. Ainsi, il n'est pas rare de rencontrer des propriétaires ayant commandé à un pépiniériste du chêne sessile et se voyant livrer du pédonculé ! L'impact sylvicole est d'autant plus important que ces semis sont installés pour plusieurs dizaines d'années, et que les deux essences ont des auto-écologies très différentes (Becker, 1984).

Or, la différenciation des chênes sessile, pédonculé et même pubescent, peut être établie de façon nette sur le terrain, d'après les caractères de port des arbres, de morphologie foliaire et des infrutescences (Guinier, 1950; Dupouey, 1983; Grandjean et Sigaud, 1987). En effet, si les 2 espèces présentent parfois des individus morphologiquement intermédiaires, leur nombre est toujours restreint (moins de $5 \%$ de l'effectif des populations), et ils sont cantonnés à des milieux particuliers. II est donc possible, lors d'une récolte de glands, d'identifier les 2 espèces.

En revanche, il est toujours apparu très difficile de contrôler a posteriori la pureté des lots de glands fournis à l'entrée des pépinières. De nombreux travaux signalent des différences plus ou moins nettes entre chêne sessile et pédonculé, quant à la couleur : présence de stries longitudinales foncées, à l'état frais, sur le gland de chêne pédonculé, et quant à la forme.
Le gland du chêne pédonculé serait souvent plus gros, plus allongé et cylindrique que celui du chêne sessile, lui-même plus globuleux, arrondi. Moggi et Paoli (1972), à la suite de Poskin (1934), notent des différences dans le rapport longueur sur diamètre maximum, supérieur à 1,6 pour le pédonculé, inférieur pour le sessile. De même, Poskin, puis Escarre (1973) signalent une différence sur la position du diamètre maximum du gland, celui-ci étant situé plus bas chez le sessile que chez le pédonculé. Mais d'autres études concluent à l'absence de différences significatives entre les deux espèces quant à la morphologie des glands (Becker, 1972; Rushton, 1978; Brookes et Wigston, 1979; Dupouey, 1983).

Nous essayons ici de préciser ces différences morphologiques entre chêne sessile et pédonculé, sur la base d'un échantillonnage important de glands, en espérant apporter un outil de contrôle de la pureté des lots de graines de chêne.

\section{Matériel et Méthodes}

L'étude porte sur 18 provenances de chêne pédonculé, 20 provenances de chêne sessile, 5 peuplements mélangés ou intermédiaires et une provenance d'origine inconnue. Ces peuplements avaient été choisis pour la plupart dans un but indépendant de notre étude : la mise en place d'un dispositif de comparaison de provenances de chênes par le groupe "Chêne-pays de Loire". Leur liste est donnée au Tableau I, avec indication, selon le récolteur, de la nature, sessile ou pédonculée, de l'espèce. Cette indication, laissée à l'appréciation du récolteur, peut être sujette à caution. II est à noter, comme c'est malheureusement le cas pour de telles récoltes, que la surface échantillonnée et la densité de l'échantillonnage au sein de cette surface peuvent varier considérablement d'un peuplement à l'autre (d'un arbre isolé à une dizaine d'hectares).

Les lots de glands sont tous issus de la glandée 1984. Les glands de faible densité (et donc 


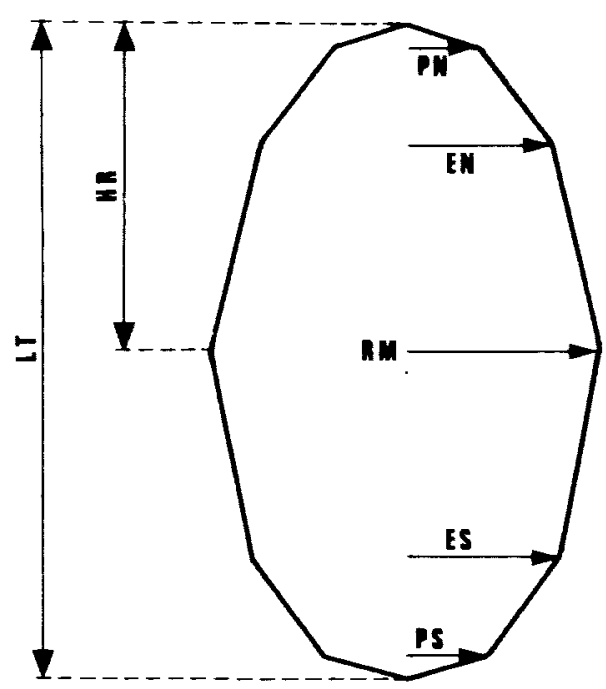

Fig. 1. Variables morphologiques mesurées.

de faible viabilité) sont éiiminés par flottation. Les mesures sont effectuées sur la photocopie de 100 glands prélevés au hasard dans chaque lot. Elles sont au nombre de sept (Fig. 1) :

- longueur totale (LT);

- rayon maximal (RM);

- distance de l'apex du gland au rayon maximal (HR);

- rayon "polaire nord" ( $P N$ ), égal au rayon mesuré à $1 \mathrm{~mm}$ de l'apex;

- rayon eéquatorial nord" (EN), mesuré à $5 \mathrm{~mm}$ de l'apex;

- rayon «équatorial sud» (ES), mesuré à $5 \mathrm{~mm}$ de la base;

— rayon "polaire sud" (PS), mesuré à $1 \mathrm{~mm}$ de la base.

On dispose, en parallèle, de la mesure du pourcentage de feuilles ayant des oreillettes développées à la base du limbe (bon caractère de discrimination des chênes sessile et pédonculé) sur les plants de un an dans les placettes où les lots de glands initiaux ont été semés. Le poids des glands est aussi noté.

L'étude de ces données est effectuée par :

- analyse en composantes principales des 4400 glands et 7 variables morphologiques afin de décrire la variabilité observée dans la forme des glands de ces provenances;
Tableau 1 . Liste des provenances de chêne étudiées. La première lettre indique l'espèce identifiée par le récolteur ( $P$ : Quercus petraea, $R$ : Quercus robur, $M$ : mélange ou intermédiaire).

\begin{tabular}{lll}
\hline \multicolumn{2}{c}{ Forêt } & Département \\
\hline Quercus robur & \\
R01 & Saint-Martin-du-Bois & Maine-et-Loire \\
R02 & Vezins & Maine-et-Loire \\
R03 & Pontménard & Maine-et-Loire \\
R04 & Le Luart & Sarthe \\
R05 & La Pierre & Sarthe \\
R06 & Les Viennières & Mayenne \\
R07 & Charnie & Mayenne \\
R08 & Aire-sur-l'Adour & Landes \\
R09 & Ognoas & Landes \\
R10 & Bastennes & Landes \\
R11 & Mées & Landes \\
R12 & Inconnu & Hautes-Pyrénées \\
R13 & Inconnu & Hautes-Pyrénées \\
R14 & Inconnu & Hautes-Pyrénées \\
R15 & Seillon & Ain \\
R16 & Colonne & Jura \\
R17 & Dole & Jura \\
R18 & Dijon & Côte-d'Or \\
& & \\
Quercus petraea & \\
P01 & Le Poinçonnet & Indre \\
P02 & Chandelais & Maine-et-Loire \\
P03 & Monnaie & Maine-et-Loire \\
P04 & Teillay & Loire-Atlantique \\
P05 & Juigné & Loire-Atlantique \\
P06 & Escoublac & Loire-Atlantique \\
P07 & Le Gavre & Loire-Atlantique \\
P08 & Le Gavre & Loire-Atlantique \\
P09 & Le Gavre & Loire-Atlantique \\
P10 & Le Gavre & Loire-Atlantique \\
P11 & Vouvant & Vendée \\
P12 & Vibraye & Sarthe \\
P13 & Vibraye & Sarthe \\
P14 & Sillé & Sarthe \\
P15 & Sillé & Sarthe \\
P16 & Perseigne & Sarthe \\
P17 & Bellebranche & Mayenne \\
P18 & Bourgon & Mayenne \\
P19 & Grande Charnie & Mayenne \\
P20 & Les Effretais & Mayenne \\
& & \\
Peuplements mélangés ou intermédiaires \\
M01 & Carquefou & Loire-Atlantique \\
M02 & Vibraye & Sarthe \\
M03 & Monnaie & Maine-et-Loire \\
M04 & La Proutière & Vendée \\
M05 & Fontainebleau & Seine-et-Marne \\
INC & Inconnue & inconnu \\
& & \\
\hline
\end{tabular}


- analyse factorielle discriminante de ces provenances selon le critère espèce, afin de déterminer la forme caractéristique des glands de chacune des deux essences.

\section{Résultats}

L'analyse en composantes principales montre la présence de deux axes de variations de la morphologie au sein de l'ensemble des glands mesurés, totalisant $80 \%$ de la variance totale. Le premier (52\% de la variance) représente la variation du diamètre du gland (variables RM, PN, EN, PS, ES). Le second axe (27\% de la variance), correspond aux variations de longueur du gland ( $L T$ et HR). Un troisième axe ( $10 \%$ de la variance totale) oppose les variables $P N$ et $P S$, donc les glands cylindriques aux glands ogivaux.

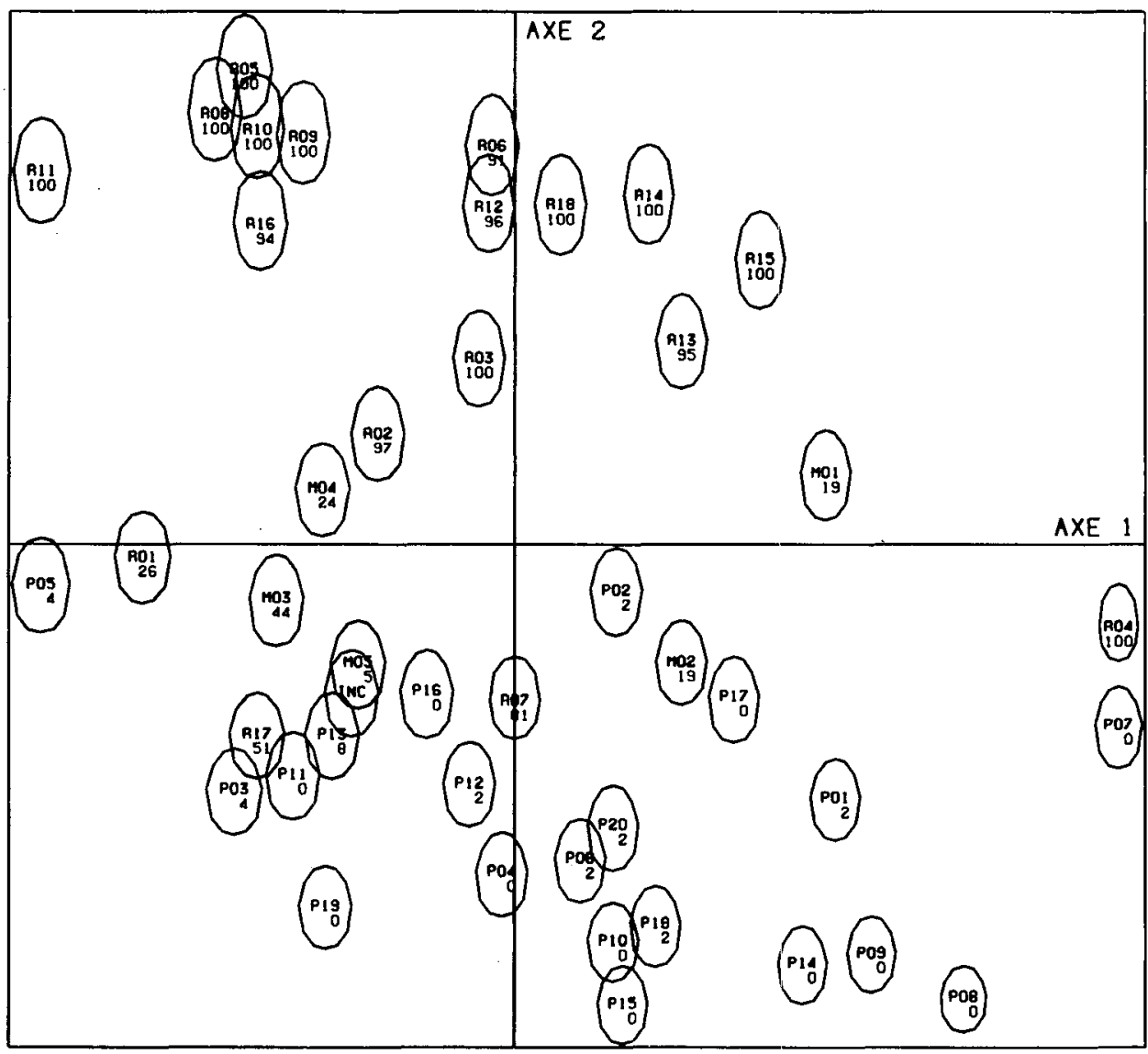

Fig. 2. Plan des axes 1 et 2 de l'analyse en composantes principales des 4400 glands et 7 variables morphologiques. On a représenté le point moyen de chaque provenance avec le pourcentage de feuilles auriculées observé dans la provenance. 
Si l'on représente le point moyen de chaque provenance sur le plan des axes 1 et 2 de la projection des individus (Fig. 2), on constate une très bonne séparation des provenances sessile et pédonculée au long du second axe, donc selon la longueur du gland. Un faible décalage apparaît aussi sur l'axe 1 , selon le diamètre. Ainsi, une part importante de la variabilité morphologique du gland est liée à l'espèce, par la longueur du gland ou la distance de son diamètre maximal à l'apex, et, dans une moindre mesure, par son diamètre.

Le pourcentage d'oreillettes observé sur les plants de un an dans ces mêmes provenances est très lié lui aussi à la forme du gland : à chaque extrémité de l'axe 2, les pourcentages d'oreillettes sont ou tous nuls (chêne sessile), ou tous proches de $100 \%$ (chêne pédonculé). Par contre, les provenances qui apparaissent en position intermédiaire sur l'analyse sont aussi celles ayant un pourcentage d'oreillettes non caractéristique : M01, M02, M03, M04, R01, R07 et R17. Pour ces provenances, entre 20 et $80 \%$ de plants ont des feuilles auriculées. Les provenances P05 (glands très larges) et R04 (glands très étroits) s'isolent aux deux extrémités de l'axe 1.

On observe sur le graphique le regroupement de quelques provenances géographiquement proches. R08, R09, R10 et R11, 4 provenances du bassin inférieur de l'Adour sont morphologiquement très semblables, de même que R12, R13 et R14, toutes 3 des Hautes-Pyrénées. Parmi les chênes sessiles, P07 à $\mathrm{P} 10$ proviennent de la même forêt (le Gavre, Loire-Atlantique) et P14, P15, P18, P19 sont trois provenances proches (confins de la Mayenne et de la Sarthe). Notons aussi P02 et M03, deux provenances de la Sarthe ainsi que P12, P13 et M02 de la forêt de Vibraye (Sarthe).
Tableau II. Valeur moyenne des variables mesurées pour chaque espèce; différence significative au seuil de $5 \%:$ :; $1 \%$ : ${ }^{\star \star}$; $1 \%:{ }^{\star \star *}$.

\begin{tabular}{|c|c|c|c|}
\hline & $\begin{array}{c}\text { Quercus } \\
\text { robur }\end{array}$ & $\begin{array}{l}\text { Quercus } \\
\text { petraea }\end{array}$ & $\begin{array}{c}\text { Test de } \\
\text { la différence }\end{array}$ \\
\hline $\mathrm{LT}(\mathrm{mm})$ & 29,5 & 25 & $* * *$ \\
\hline $\mathrm{RM}(\mathrm{mm})$ & 7,9 & 7,8 & ** \\
\hline $\mathrm{HR}(\mathrm{mm})$ & 15,1 & 11,7 & $\star \star \star \star$ \\
\hline $\mathrm{PN}(\mathrm{mm})$ & 3 & 3,2 & 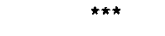 \\
\hline $\mathrm{EN}(\mathrm{mm})$ & 6 & 6 & NS \\
\hline $\mathrm{ES}(\mathrm{mm})$ & 6,3 & 6,5 & $* * *$ \\
\hline PS (mm) & 3,4 & 3,5 & * \\
\hline$P O(g)$ & 45 & 34 & $\star \star \star *$ \\
\hline
\end{tabular}

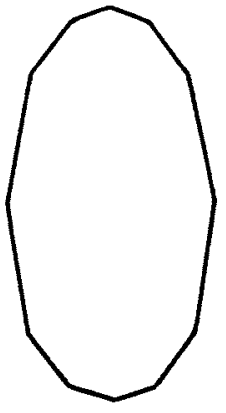

Quercus robur

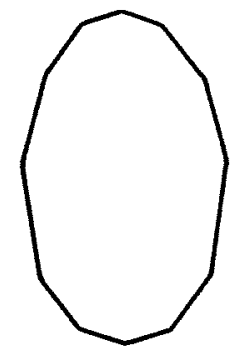

Quercus petroea
Fig. 3. Forme moyenne des glands de chêne sessile et pédonculé, à l'échelle.

Après élimination des peuplements douteux (entre 20 et $80 \%$ de feuilles auriculées), on peut caractériser chaque espèce. Le Tableau II donne les valeurs moyennes pour chaque variable et le seuil de signification de la différence. Seul le rayon équatorial nord n'apparaît pas différent entre les deux espèces. La Figure 3 montre la forme moyenne du gland de chaque espèce, à l'échelle. L'analyse discriminante de ces 2 ensembles de glands permet de déterminer les variables morphologiques séparant au mieux les 2 espèces, et de fournir une formule discriminante. 
Comme on pouvait s'y attendre au vu des résultats de l'analyse en composantes principales, les 2 variables les plus discriminantes sont, successivement, la distance du rayon maximal à l'apex, puis le rayon maximal. L'introduction d'autres variables explicatives n'améliore pas le modèle obtenu puisqu'elles sont toutes fortement corrélées à ces deux premières variables.

L'équation discriminante obtenue est :

$$
\text { 112. } \mathrm{HR}-84 . \mathrm{RM}-843
$$

(HR et RM exprimés en $\mathrm{mm}$ )

Elle fournit un indice d'espèce dont les valeurs positives indiquent un gland de chêne pédonculé, les valeurs négatives un gland de chêne sessile. Le pourcentage de glands bien classés par cette équation est de $80 \%$ pour le chêne pédonculé et $88 \%$ pour le chêne sessile.

\section{Discussion - conclusion}

Nos résultats confirment certaines observations antérieures, plus ou moins subjectives, sur la base d'un échantillonnage plus important et par des mesures quantitatives. On obtient ainsi une bonne séparation des deux espèces en fonction de la morphologie de leur graine. La différence de rapport longueur sur diamètre maximum observée par Poskin (1934) (1,7 chez le chêne pédonculé et $1,5 \mathrm{chez}$ le sessile) se retrouve ici, avec des valeurs moyennes légèrement supérieures $(1,9$ pour le pédonculé, 1,6 pour le sessile). Poskin obtenait avec ce seul rapport des taux de discrimination de $66 \%$ pour le pédonculé et de $70 \%$ pour le sessile. L'utilisation d'une fonction affine du rayon maximum et de sa position sur le gland permet d'augmenter sensiblement ces taux. Par contre, la différence de hauteur relative du diamètre maximum observée par le même auteur ne se retrouve pas : le diamètre maximum s'établit à la moitié de la hauteur sur tous les glands.

Notre échantillon est toutefois biaisé par une surreprésentation des peuplements de chêne sessile de l'ouest et de chêne pédonculé du bassin de l'Adour. Ce déséquilibre peut être de nature à limiter la portée des résultats, en particulier la validité hors de ces zones de la fonction discriminante proposée. L'étude devrait donc être étendue à la moitié est de la France. La caractérisation des peuplements récoltés devra être faite, à cette occasion, non pas a posteriori sur la morphologie foliaire de plants issus des glands récoltés, mais, de façon beaucoup plus sûre, par la mesure de la longueur moyenne des pédoncules des infrutescences des individus échantillonnés. Notons d'ores et déjà que Poskin (1934), Escarre (1973), Moggi et Paoli (1972) ont obtenu des résultats concordants dans des régions très différentes de la nôtre (respectivement la Belgique, l'Espagne et I'Italie).

De même, les récoltes ne concernent qu'une seule année, et on peut penser qu'un facteur climatique global influence la forme générale (en particulier la taille) des glands. De façon à pouvoir utiliser pratiquement l'équation discriminante, il serait bon de la recalculer sur plusieurs années sur des provenances bien identifiées, afin de vérifier sa stabilité.

Enfin, la discrimination obtenue l'a été sur des glands récoltés in situ. Les caractères morphologiques observés peuvent donc être aussi bien d'origine génétique (effets espèce, provenance, descendance...) que phénotypique, c'est-à-dire liés au climat particulier de chaque région de provenance, à son substrat géologique ou au type de station de récolte. Seules, en théorie, des plantations comparatives permettraient de s'affranchir de l'influence du 


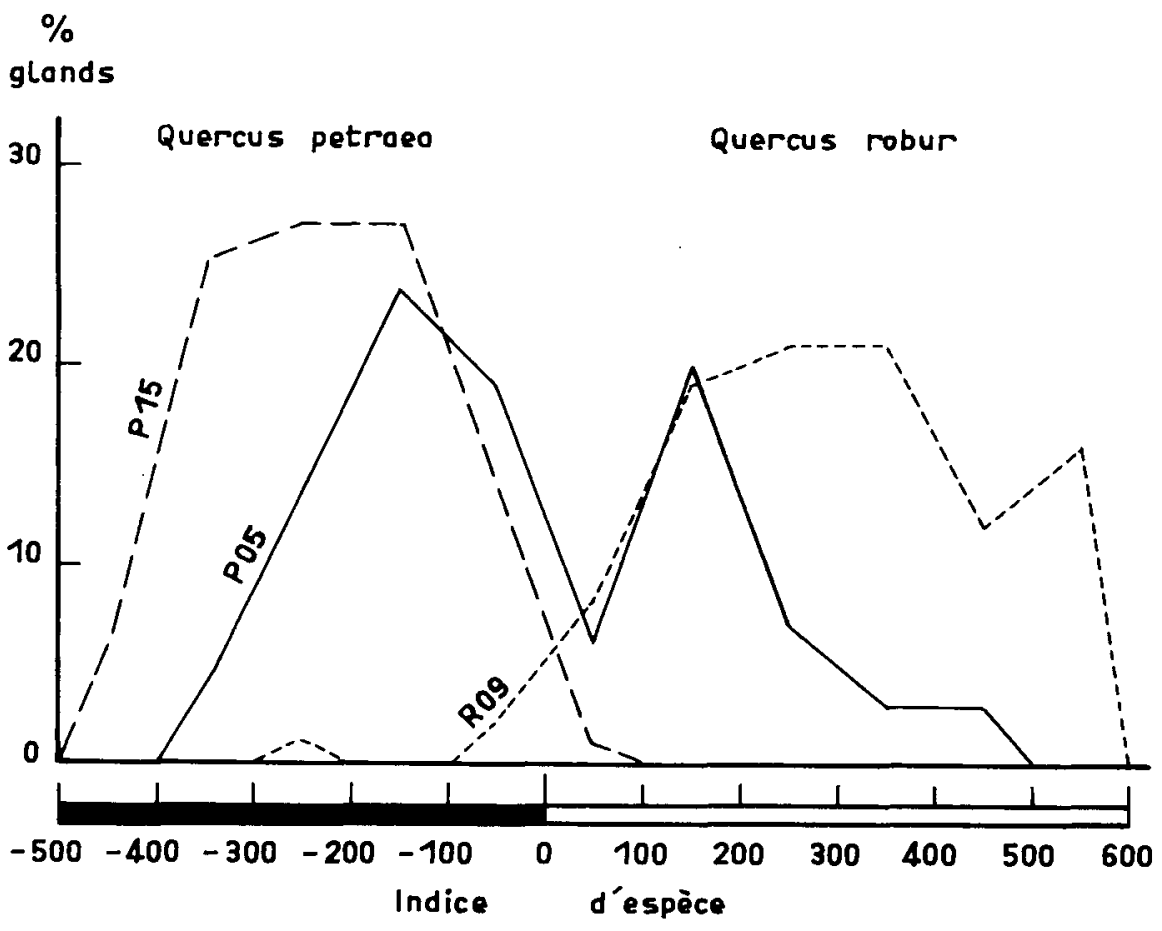

Fig. 4. Histogrammes de l'indice spécifique pour 3 provenances.

milieu. Mais on peut tout de même constater que les variations observées en fonction de l'espèce restent valables quelles que soient les régions, et ont donc certainement une large part d'origine génétique.

La fonction discriminante donne une proportion relativement importante de graines mal classées (15\% en moyenne), au niveau individuel. L'utilisation de marqueurs génétiques plus performants, telles les iso-enzymes, devrait permettre, à condition de découvrir des marqueurs spécifiques, d'atteindre des taux de reconnaissance proches de $100 \%$, mais à des coûts bien sûr très supérieurs. II faut cependant noter que notre méthode, si elle ne garantit pas dans tous les cas une identification certaine de l'espèce au niveau gland individuel, permet, avec la mesure de 100 glands, une bonne carac- térisation des provenances. On trouvera en Figure 4 la courbe de répartition de l'indice d'espèce calculé pour les 100 glands de 3 provenances : pédonculé typique (R09), sessile typique (P15), et intermédiaire (P05). On observe pour celle-ci une répartition bimodale de l'indice spécifique, avec une prédominance des formes de type sessile dans l'échantillon. On peut suspecter dans ce cas un mélange des 2 espèces dans le peuplement de récolte.

D'un point de vue physiologique, le poids plus important de la graine de chêne pédonculé $(30 \%$ de plus que celle de chêne sessile) peut en partie expliquer la production plus importante en biomasse des jeunes plants de pédonculé, au moins jusqu'à la mise en place du premier étage foliaire (Lamond, 1978). 
Nos résultats permettent d'envisager des techniques pratiques de tri des glands à l'entrée des pépinières ou le contrôle de la pureté d'un lot de semences donné.

\section{Références}

Becker M. (1972) Quelques observations morphologiques chez le chêne sessile et le chêne pédonculé. Bull. Soc. Bot. Fr. 119, 3-4, 231-236

Becker M. (1984) A propos du dépérissement du chêne : réflexion sur la place actuelle de cette espèce dans la forêt française. Rev. Géogr. Pyrénées Sud-Ouest 55, 2, 173-180

Brookes P.C. \& Wigston D.L. (1979) Variation of morphological and chemical characteristics of acorns from populations of Quercus petraea (Matt.) Liebl., $Q$. robur L. and their hybrids. Watsonia 12, 315-324

Dupouey J.L. (1983) Analyse multivariable de quelques caractères morphologiques de populations de chêne (Quercus robur L. et Quercus petraea (Matt.) Liebl) du Hurepoix. Ann. Sci. For. (Paris) 40, 3, 265-282

Escarre A. (1973) Introduccion a la taxonomia numerica de los Quercus. Estudio de la estruc- tura sistematica y espacial del robledal del valle de la Burunda. Thèse doctorat, université de Barcelone

Grandjean G. \& Sigaud P. (1987) Contribution à la taxonomie et à l'écologie des chênes du Berry. Ann. Sci. For. (Paris) 44, 1, 35-66

Guinier P. (1950) Qu'est-ce que le Chêne ? Bull. Soc. For. Franche-Comté Provinces de l'Est 25, 553-589

Lamond M. (1978) Influence des cotylédons sur la croissance et le développement du système racinaire du chêne pédonculé (Quercus robur L.). Symposium "Root Physiology and Symbiosis", Nancy, 11-15 sept. 1978 (Riedacker A \& Gagnaire-Michard J., Eds) IUFRO, pp. 228241

Moggi G. \& Paoli P. (1972) Ricerche sulle Querce caducifoglie italiane. 1 - Sul valore di alcuni caratteri biometrici e morfologici. Webbia 26, 2, 417-461

Poskin A. (1934) Le Chêne Pédonculé et Le Chêne Rouvre, leur Culture en Belgique. Ed. Duculot, Gembloux

Rushton B.S. (1978) Quercus robur L. and Quercus petraea (Matt.) Liebl. : a multivariate approach to the hybrid problem, 1. Data acquisition, analysis and interpretation. Watsonia 12 , $81-101$ 